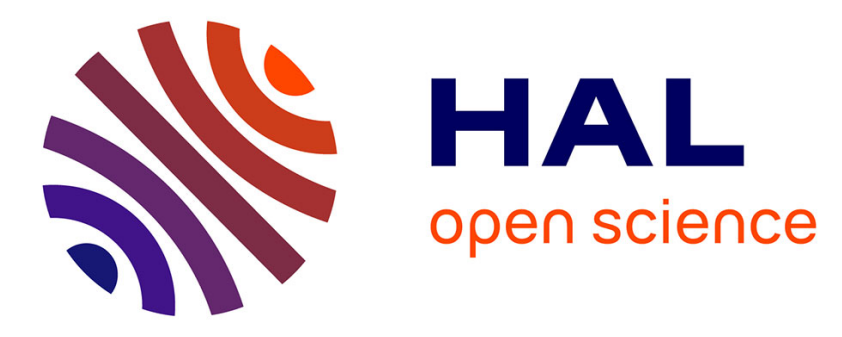

\title{
Exploring Interactions in Vehicular Networks
}

Felipe Domingos da Cunha, Davidysson Abreu Alvarenga, Guilherme Maia, Aline Carneiro Viana, Raquel A. F. Mini, Antonio A. F. Loureiro

\section{To cite this version:}

Felipe Domingos da Cunha, Davidysson Abreu Alvarenga, Guilherme Maia, Aline Carneiro Viana, Raquel A. F. Mini, et al.. Exploring Interactions in Vehicular Networks. MobiWac 2016 - 14th ACM International Symposium on Mobility Management and Wireless Access, Nov 2016, Malte, Malta. hal-01369341

\section{HAL Id: hal-01369341 https://hal.inria.fr/hal-01369341}

Submitted on 21 Sep 2016

HAL is a multi-disciplinary open access archive for the deposit and dissemination of scientific research documents, whether they are published or not. The documents may come from teaching and research institutions in France or abroad, or from public or private research centers.
L'archive ouverte pluridisciplinaire HAL, est destinée au dépôt et à la diffusion de documents scientifiques de niveau recherche, publiés ou non, émanant des établissements d'enseignement et de recherche français ou étrangers, des laboratoires publics ou privés. 


\section{Exploring Interactions in Vehicular Networks}

\author{
Felipe D. Cunha \\ DCC - UFMG \\ fdcunha@dcc.ufmg.br \\ Aline C. Viana \\ INRIA - Saclay \\ aline.viana@inria.fr
}

\author{
Davidysson A. Alvarenga \\ DCC - PUC Minas \\ davidysson@ufmg.br \\ Raquel A. F. Mini \\ DCC - PUC Minas \\ raquelmini@pucminas.br
}

\author{
Guilherme Maia \\ DCC - UFMG \\ jgmm@dcc.ufmg.br \\ Antonio A. F. Loureiro \\ DCC - UFMG \\ loureiro@dcc.ufmg.br
}

\begin{abstract}
Vehicular networks are networks comprised of vehicles traveling cities and highways. During their trajectories, these vehicles interact with other vehicles and roadside units, to make safer and enjoyable traffic. Several factors may influence these interactions. To mention a few: vehicle speed, roads condition, time of day and weather. Moreover, driver behavior and its interests can influence in vehicle features. In this context, the Vehicular Social Networks arise as a new perspective to vehicular networks, where the vehicles "socialize" and share common interests. In this work, we check the behavior of vehicles using two mobility scenarios, to classify them according to the interactions performed, identifying common interests and similar routines. Thus, we use metrics of complex networks and statistical techniques. Results prove the existence of routines and human features in Vehicular Networks.
\end{abstract}

\section{Categories and Subject Descriptors}

C.2.0 [Computer-Communications Networks]: GeneralData communications; C.2.1 [Computer-Communications Networks]: Network Architecture and Design-Wireless communication

\section{Keywords}

Vehicular Networks Interactions Social Behaviors Data Science

\section{INTRODUCTION}

Vehicular Networks are a type of network comprised of vehicles that have communication capacity, which enables them to interact each other directly or through infrastructure placed on public roads. These vehicles also have processing capacity and sensing. However, due to the high vehicle mobility, these networks present a high variation in its topology. Other features can influence to this variation are the speed limits and the direction imposed by public roads.
Many are the factors that can affect these interactions. To mention a few: the time of day, vehicle speed, location, traffic jams, events, weather, etc. For example, in rush hours (dense traffic), public roads can be seen as a popular place, where it is more susceptible occur interactions. Besides, the trajectories are also closely related to the drivers' behaviors, since their routines influence the places they visit and the periods of the day those visits occur. This brings a new perspective to vehicular networks: Vehicular Social Networks - VSNs [18].

In this context, this study aims to investigate the VSNs, studying the influence of human behavior in vehicular mobility, identifying patterns, similar routines, and common interests. In addition, we discuss the improvements that this knowledge can offer to the establishment of vehicular networks. In our previous work [4], we presented a study of the interactions using taxi mobility traces, where we modeled it as temporal graphs computing metrics to identify features and behaviors. In a different way, in this work we use a real trace that portrays a taxi mobility in Rome city and synthetic trace derived from the "working day model" [6] that describes the people mobility in Helsinki city. For this, we analyzed the traces using metrics of complex networks and statistical techniques highlighting the behaviors and features. The results show that it is possible to identify classes of behavior in social networks vehicle.

The remainder of this work is organized as follows. In Section 2, we present a brief survey of the related work. In Section 3, we describe the traces' features and the methodology used to analyze the vehicular interactions. In Section 4, we discuss the numerical results and the correlation among the metrics analyzed. In Section 5 we present some perspectives to apply interactions to promote new services and protocols in VANETs. Finally, in Section 6, we present the final remarks and future works.

\section{RELATED WORK}

"Computer networks are inherently social networks, linking people, organizations, and knowledge" [20]. In this context, different kinds of computer networks use to explore these social concepts in order to better understand the topology network evolution and to analyze nodes' encounters. The main objective is to use these concepts is to improve the performance of network protocols.

In vehicular networks, due to their particularities, it is interesting to execute traffic mobility analysis aiming to improve the protocols and services. In [7], the authors present a depth analysis of the topological properties of vehicular 
networks. They explored the social metrics in different mobility models, using social metrics to investigate the temporal evolution of the network topology. Results show new perspectives to take advantage of the vehicular mobility to improve the network protocols. Differently, of our work, this study is based only on analytical mobility models.

On the other hand, in [14] and [11], the authors analyzed and realistic mobility traces. They discuss how social metrics can be applied to improve the performance of the routing protocols in VANETs. The results show the importance of considering topology aspects to design the new protocols. However, the analysis does not consider the whole dataset, it is based just on a period of 2 hours. Likewise, in [10] the authors discuss several universal laws of social networks, presenting an investigation of two dataset traces. Nevertheless, in their analysis they only consider a reduced number of vehicles and thy compute three social metrics, i.e., node degree, distance, and cluster coefficient.

Continuing the study of vehicular mobility, in [17], it is presented a macro and microscopic study of a synthetic vehicular trace. The macroscopic study comprises the evolution of vehicular density and the dynamic of large-scale vehicles flows, and the microscopic study considers the vehicles distribution in the area and the contact among them. The authors present the traffic evolution over the area and time, discussing the importance of designing protocols and services to VANETs considering the dynamism of the traffic.

In the same context, the study of the dynamic networks is presented in [5]. In this work, the authors present a strategy to evaluate users' interactions. They define a classification to interactions between random and social relationships. Similarly, the authors evaluated mobility traces that portray the mobility of different user's type. However, the analysis is performed using a long slot time, grouping all encounters that happen during one day. In our work, we consider all days of the trace, where we figure out how the interactions happen among the vehicles, time and environment.

Applying the social concepts, in [12] the authors discuss the new perspectives of the vehicular social networks, presenting an analysis of the relevant works published. They discuss the major social properties that can be used in the design of vehicular communication systems, specifically the centrality and the community. Also, they present the framework SocialDrive, an online social strategy for vehicles transiting in platoons able of publishing dynamic context and to subscribe using online context-aware filters. The results showed that this strategy helps to enhance the social cooperative communication between drivers.

\section{METHODOLOGY}

In this Section, we define the concepts used to perform the vehicular mobility analysis. It was divided into three parts: (i) we present the characteristics of mobility traces chosen to be evaluated in this study (Section 3.1); (ii) we define the model used to create the temporal graphs and the random graphs based on the encounters among the vehicles (Section 3.2); (iii) we describe the complex networks metrics computed on the graphs, highlighting the proprieties measured for each one (Section 3.3).

\subsection{Temporal Graphs}

The temporal graphs are created take into account the unit disc model, which allows us to make the inference of the encounter between vehicles, i.e., when two vehicles are within communication range of each other. In our evaluation, we consider the communication range of $100 \mathrm{~m}$, according to the communication protocol 802.11p.

Then, we map the vehicular mobility and encounters described in the trace into a temporal graph. We insert an edge between two nodes (vehicles) when we have an encounter. The Figure 1 presents the number of components and the edge density to the graph to the Rome and Helsinki trace, when we range of the time slot duration. As we can see, very short time slot implies in a fragmented network, presenting a lot of nodes without connections and low-density graph. In this scenario, we have a lot of nodes without connections. On the other hand, long time slot generates a small number of components and higher density graph, which portrays a homogeneous behavior in the metrics. In this case, the graphs present the structure similar to a complete graph. Thus, we divide the whole trace into discrete time slots of duration $t=15 \mathrm{~min}$, aiming to better capture the traffic changes and the dynamics of the network. We generate each temporal graph $G(t)$ using the Growing Time Window technique [8], which puts together all of the encounters which happened during the same time slot. All the results consider this time slot in the metrics evaluation.

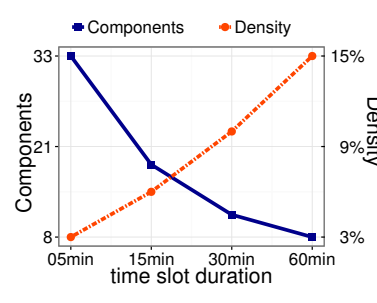

(a) Rome

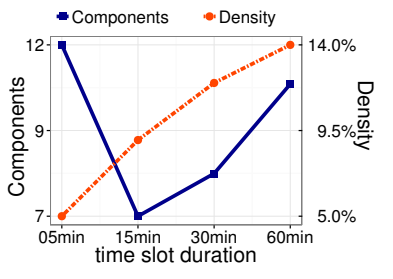

(b) Helsinki
Figure 1: Number of Components and Edge Density varying time slot window for $\mathbf{1 0 0}$ meters of communication range.

The temporal graph at the time $t$ is a undirected graph, and can be formally defined as a graph $G(t)=(V, E)$, where $V$ represents the set with all vehicles $v_{i}$ and $E$ represents the set of edges $e_{i j}$. In $G(t)$, an edge $e_{i j}(t)$ exists between the vehicles $v_{i}$ and $v_{j}$ during the time slot $t$, with $i \neq j$. The evaluation of each metric is applied in each graph, taking into account each temporal graph $G(t)$. Thus, we intend to analyze the encounters that happen in each period $t$. For each trace, 96 graphs are generated describing the vehicles encounters per day $G(t)=\left\{G\left(t_{1}\right), G\left(t_{2}\right), G\left(t_{3}\right), \ldots, G\left(t_{96}\right)\right\}$. The final metrics value represents the average for each metric in each time slot analyzed. Also, in order to verify the structures on the graph and the relationship among the vehicles, we compare the results with random graphs $G^{R}$ generated with the same degree distribution. In random graphs, the process to insert an edge is probabilistic and this graph has a different structure than the temporal graphs.

In this work, a random graph $G^{R}$ is a graph obtained from 
a set of nodes and its respective degree distribution. The criteria to add an edge between two nodes in the graph $G^{R}$ is made in a random way, based on probabilities models. The model used to add edges in this work uses the urn algorithm that creates random structures presented in [9]. Firstly, this algorithm inserts $d_{i}$ balls in an urn, marked with the identifier $i$ of the node. A number of balls of each node is equal to the node degree. The total amount of balls in the insertion process in the urn, matches with the degree distribution of the temporal graph $G(t)$. Since this step, two balls $b_{i}$ and $b_{j}$ are randomly selected and their identifiers are compared. If $i \neq j$ and there are no edge between these nodes, an edge $e_{i j}$ is added. The selection step is made until there is no ball inside the urn, or there is no possibility to add more edges in the graph.

\subsection{Traces}

Traces are a special dataset type that contains information about the trajectory of vehicles, with the purpose of tracking the vehicles' mobility during a trajectory. Traces are very good dataset since they portray the vehicular behavior in a certain scenario. Normally, in literature, there are two trace types: real and realistic. Real traces have the information collected from a group of vehicles, with the use of some localization system device (e.g. GPS); and realistic traces are created by the junction of maps of a particular locality, historic of traffic in this locality, statistics models, and a mobility simulator. Following, we describe the main features of the two traces used in our evaluation.

Rome: This real trace contains the mobility trace of taxi cabs in Rome, Italy. Each taxi vehicle has a GPS receiver and a tablet that periodically ( 7 seconds) retrieves the GPS position and sends it to a central server. The trace contains GPS coordinates of approximately 320 taxis, collected over 30 days in the Rome area, from February 01 to March 02 of 2014 [2]. However, traces can present spatial and temporal gaps. In [16] the authors present a technique to fill these gaps. In this work, we apply this technique in the Rome trace, filling the spatial gaps aiming to enrich the dataset.

Helsinki: This data set is created based on the model "working day model" [6]. This model defines the nodes mobility according to three different major activities that the nodes can be doing. They are being at home, working and some evening activity with friends. These activities are the most common and capture most of a working day for the majority of people. The activities differ from each other. These sub-models repeat every day, resulting in periodic repetitive movement. Their configuration allows fine-tuning the model to meet the needs of the target scenarios. Communities and social relationships are formed when a set of nodes are doing the same activity in the same location.

Nodes are doing the activities on a daily basis starting from home in the morning. Each node is assigned a wake-up time, which determines when the node should start from home. The variance in wake-up time models the differences in times in real life. At wake-up time, nodes leave their homes and use different transport methods to travel to work. Nodes travel between activities either by car or by bus, which are both different sub-models. [6].

Based on this model, we generate the mobility of 2.000 people taking different transport types such as car, buses, and taxis. These people move in 3 different districts (home, work and leisure points), every day during one week. The

\begin{tabular}{lc} 
Parameters & Values \\
\hline \# of people & 2 mil \\
\hline Groups & 8 groups in 3 districts \\
\hline$\%$ of vehicles & $50 \%$ \\
\hline Places & Home, work and leisure points \\
\hline Transport Type & Buses, cars and taxis \\
\hline
\end{tabular}

Table 1: Working Day Movement Model parameters.

traffic simulation is made on The ONE simulator, using the parameters described on Table 1 and the dataset resulted from this simulation is analyzed.

\subsection{Metrics Evaluated}

In this section, we describe the metrics chosen for this evaluation, considering the aspects and characteristics of the vehicular mobility. As for vehicles mobility, we classify these metrics in macroscopic and microscopic metrics according to the behaviors presented in [17]. The macroscopic metrics portray measures of the network global state, which can represent the general behavior of all vehicles and the temporal graph evolution. The microscopic metrics define individual values for the vehicle representing the behavior of a unique vehicle. We select as macroscopic metrics the Distance, Density, and Edge Persistence, and as microscopic metrics, we select the Node Degree, Betweenness, Cluster Coefficient, and Topological Overlap.

\section{Macroscopic Metrics:}

Distance: this is a metric that comprises the length of a path between a pair of vehicles considering the number of hops between them. In VANETs, the distance can represent the existence of common interest among the drivers. Vehicles that visit close places or have congruent trajectories have short distance, and vehicles physically separated or vehicles that transit in different areas have long distance. Considering the small work phenomenon, the graphs present short distances.

Density: this metric represents how dense is the network, i.e., the edges ratio between the vehicles. In the context of VANETs, urban regions can have higher densities than rural areas. In the downtown region, traffic jams and slow traffic happen coopering to generate higher densities. We compute this metric by the ratio between the number of existing edges $e(t)$ in the graph and the number of edges in a complete graph $|V||V-1| / 2$.

Edge persistence: is a metric that represents the persistence of an encounter among two vehicles. We consider the edge persistence as the number of times the vehicles $v_{i}$ and $v_{j}$ encounter in the same slot time in different days. We compute this metric in function of the times that this encounter $e_{i j}(t)$ happens.

\section{Microscopic Metrics:}

Node Degree: determines the number of distinct contacts that a vehicle performs during a slot time. This metric can be influenced by the vehicle' trajectory and the day period. If a vehicle passes through a higher density traffic region or a rush hour, its degree tends to be high. Also, vehicles like taxi and buses tend to present higher degree, due to the fact 


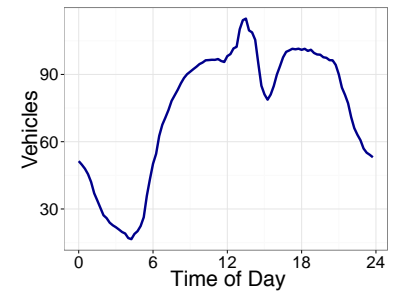

(a) Rome daily

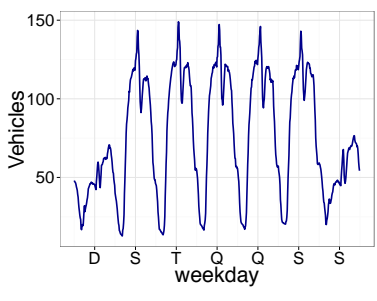

(b) Rome weekly

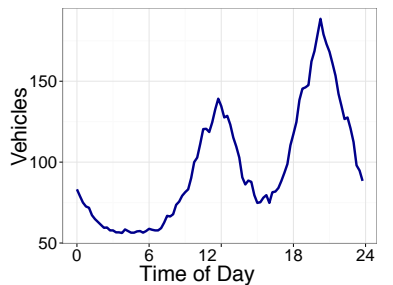

(c) Helsinki daily

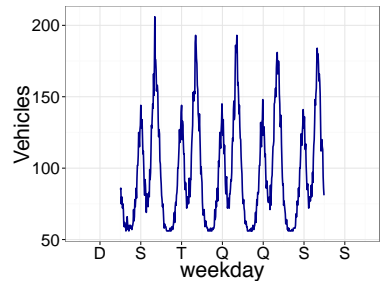

(d) Helsinki weekly

Figure 2: Traffic evolution over the day and week, in two cities: Rome and Helsinki.

that they travel many times during the day. In another hand, in regions far from the downtown or in hours with low traffic, its degree can be low or null. As a formal definition, a vehicle $v_{i}$ at slot time $t$ is defined by: Degree $_{i}(t)=\left\|\left\{v_{j} \mid \exists e_{i j}(t)\right\}\right\|$.

Betweenness: this is a metric that portrays the node's centrality in the network. Its evaluation corresponds to the number of shortest paths from all vertices to all others that cross through that node. A node that portrays high betweenness has a large influence on the network. It can be used to reach all of the other nodes quickly.

Cluster Coefficient: this metric evaluates how close are the neighbors of a vehicle $v_{i}$, i.e., the probability that two neighbors of $v_{i}$ have already encounter. This metric can represent regions with a traffic jam, describing a high cluster coefficient. Also, vehicles with common interest present higher clustering coefficient, because they share same neighbors. Besides, if the network presents the small world phenomenon, when we compare the results with the random graphs, its cluster coefficient will also be elevated [19].

Topological Overlap: this metrics portrays the similarity of contacts among the vehicles on the graph. We can define the topological overlap $t o\left(v_{i}, v_{j}\right)$ of a pair of vehicles $v_{i}$ and $v_{j}$ as the ratio of neighbors shared by two vehicles. The Equation 1 formally defines the topological overlap between the vehicles $v_{i}$ and $v_{j}[5]$.

$$
t o\left(v_{i}, v_{j}\right)=\frac{\left|\left\{k \mid\left(v_{i}, v_{k}\right) \in E_{t}\right\} \cap\left\{k \mid\left(v_{j}, k\right) \in E_{t}\right\}\right|}{\left|\left\{k \mid\left(v_{i}, k\right) \in E_{t}\right\} \cup\left\{k \mid\left(v_{j}, k\right) \in E_{t}\right\}\right|}
$$

\section{RESULTS}

In this section, we present the numerical results of the interactions characterization. This analysis was executed considering the traffic variation during the day and week. The focus is to identify behaviors and proprieties that describe the routines and characterize the interactions in vehicular networks. We analyzed the Rome calibrated trace and the Helsinki's Trace, which was generated by the working day model, according to the methodology defined in Section 3. Following, we present the traffic evolution and the results according to metrics and properties described in the Section 3.3.

\subsection{Traffic Evolution}

Taking into account the traffic evolution along of the day and week for all traces, in Figure 2 we can see these values to Rome and Helsinki's Trace. Comparing the weekdays and the weekends, we observed that the difference among these days in Rome (Fig. 2-(b)) is higher. We believe that this behavior happens due to local features. We can also note a variation over day breaks when the demand for trips reduces.

In Helsinki, Figure 2-(c) and (d), we highlight the presence of rush hours in weekdays, i.e., periods with higher traffic in contrast to the taxis traces. The traffic follows the routine of people defined by the working day model. Also, during weekends the model does not present traffic because these are days off.

\subsection{Vehicles vs. Neighbors}

Aiming to evaluate features of interactions among the vehicles and their neighbors, we compute the metrics node degree and community. The node degree represents the number of interactions a vehicle has during the slot time window. In the Figure 3, we can see the daily mean degree evolution for the three datasets, considering the communication range variation. In Rome, Figure 3-(a), we note a decrease in the degree during daybreak and, at $6 \mathrm{am}$., the value starts to increase, following the beginning of the day. This happens because during day breaks the number of trips suffers a reduction. In Helsinki, Figure 3-(b), we observe a constant behavior in the curves, with the mean degree close to 8 . Also, we note an increase in $6 \mathrm{pm}$, generated by the rush hours and traffic jams. Comparing the communication range variation, we observe the same behavior oh the curves in all of the traces, just a shift on the axis in the lines.

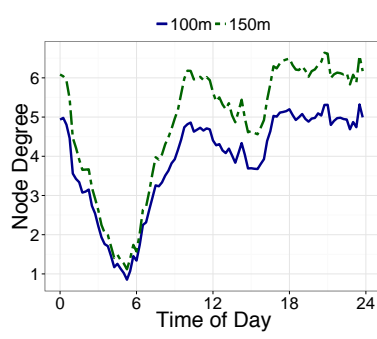

(a) Rome

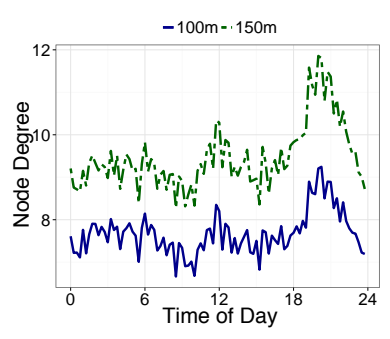

(b) Helsinki
Figure 3: Degree daily evolution for Rome and Helsinki's traces, varying the communication range.

Understanding the topology of the graphs, in Figure 4, we generate graphs (temporal and random) of each trace. In each one, we apply the walktrap community detection 


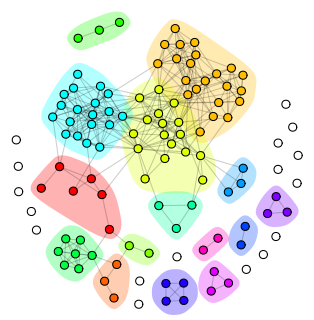

(a) Rome - $G(t)$

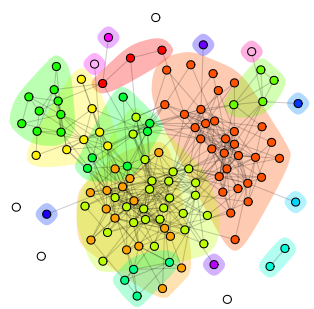

(c) Helsinki - $G(t)$

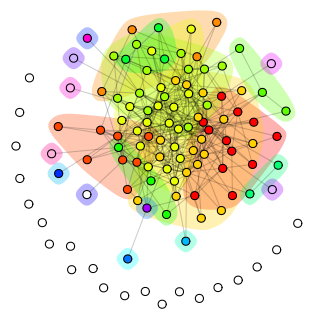

(b) Rome - $G^{R}$

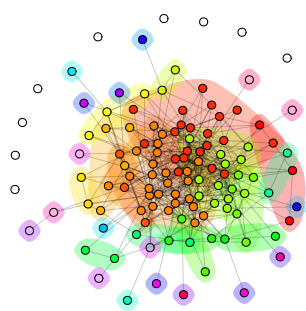

(d) Helsinki - $G^{R}$

Figure 4: Snapshot for $G(t)$ and $G^{R}$ for Rome and Helsinki's traces at $6 \mathrm{pm}$.

algorithm in order to identify the communities [13]. We can observe, comparing the real and random graphs, the presence of communities in the real graph (the nodes with the same color). All graphs, in this time, present nodes with the same interest. Taking into account the presence of communities, we can use this metric to design new services or to reach a specific community, e.g., to execute marketing and publicity or to disseminate an alert message that matters only to a specific group.

\subsection{Centrality}

Analyzing the centrality of the vehicles in the network, the Figure 5 presents the results to the mean of betweenness to all traces. Considering the criteria to add an edge on the original graph, we can observe that the random graphs reduce this value, which proves that random graphs remove the features of the original graphs. In Rome (Fig. 5-(a)), the trace presents low values to betweenness, due to the trace's characteristics where the traffic is sparser. Besides, in Helsinki (Fig. 5-(b)), the lines present constant behavior, due to the facts that in this trace we have more clusters and the vehicles transiting in same areas. Again, the random graphs reduce the values of betweenness compared to original graphs.

\subsection{Small World Phenomenon}

When we analyze the small world phenomenon in the graphs computing the distance and the clustering coefficient. The results are compared with random graphs with the same degree distribution of the original graphs. We can see in Figure 6 the average distance for both of traces during the

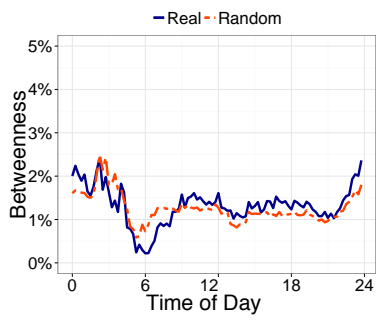

(a) Rome

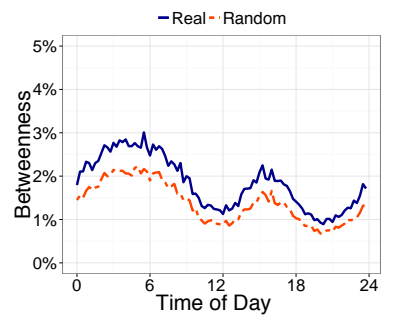

(b) Helsinki
Figure 5: Betweenness daily evolution for Rome and Helsinki's traces.

day.

As we can see in Figures 6-(a), (b), the mean distance for random graphs has a lower value compared to real traces. This happens due to the random criteria to insert an edge in a random graph. We believe that the value of distance decreases by the addition of shortcuts. For the Rome trace, Figure 6-(a), we observe that the distance follows the traffic behavior, decreasing during daybreaks. Also, considering the number of vehicles transiting, we can see that the value of the distance is shorter, close to 3 hops. Considering the Helsinki trace, Figure 6-(b), we observe a constant behavior during the day. This happens because, these values represent the average of distance, and in this trace, we have few vehicles in a short area going to same places. 


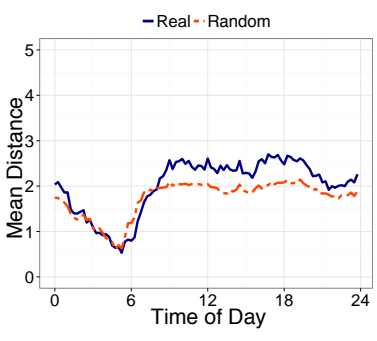

(a) Rome

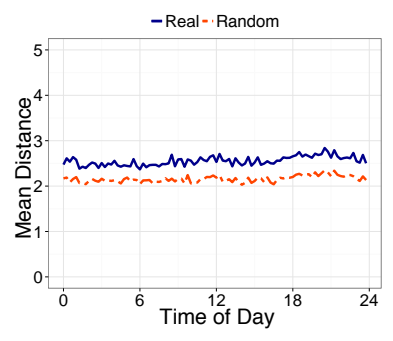

(b) Helsinki
Figure 6: Mean Distance Daily evolution for Rome and Helsinki's traces.

The results to the clustering coefficient are presented in Figure 7. Remarking this metric, we can measure how much the node neighbors are connected. Normally, the communities aggregate the nodes with the same interest. In VANETs, this behavior happens when the vehicles cross the same roads, transiting the same area.

In the Figure 7-(a), we can see the results to the Rome trace. We can note that the random graph presents an expressive difference to the real trace, which indicates that the process to generate the random $G^{R}$ breaks the communities from the temporal graph $G(t)$. Despite the traces portray the taxis' mobility, the vehicles go to the same places, guided by passengers which present similar interests. In different purpose, the Figure 7-(b) presents the values to Helsinki trace. Again, we observe a constant behavior on the lines due to the features of the trace. Beyond all, the random graph presents a result much less than the real graph, which indicates the presence of communities on the graphs.

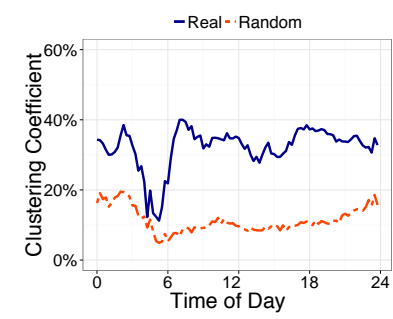

(a) Rome

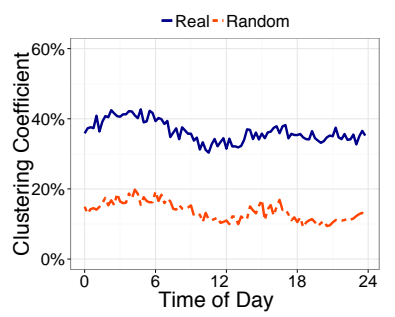

(b) Helsinki
Figure 7: Clustering Coefficient Daily evolution for Rome and Helsinki's traces.

Aiming to calculate the clustering coefficient distribution among the vehicles, in the Figure 8, we present the results of the original traces, using box plots graphics. In this Figure, we can see the values variability, the median, and the outliers for each hour. To Rome trace, we can note that during daybreak the trace presents a major variation in the values of clustering. This is expected because the traffic is sparse at this time and the destination is variable. Also, during the day, mainly in rush times, the clustering value is constant, close to $40 \%$. To Helsinki trace, we observe less variation at begin and end of the day, with values close to $40 \%$. In other hours we observe more variation, mainly during the days when the vehicles are transiting. This behavior is expected due to the features of the trace, where the people go to fixed places in same time, forming clusters.

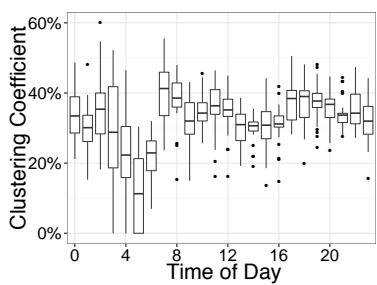

(a) Rome

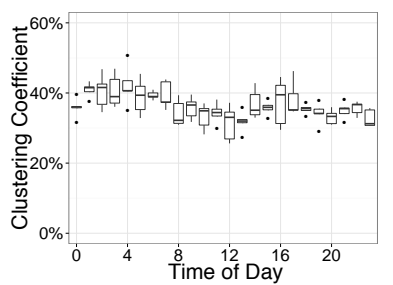

(b) Helsinki
Figure 8: Distribution for Clustering Coefficient for Rome and Helsinki's traces.

\subsection{Similar Routines}

In order to find similar behaviors on the network, we compute the metrics topological overlap and edge persistence. These are metrics that can portray similarity on the graphs. In the Figure 9, we present the values for the topological overlap during the day. In both traces, we observe that random graphs have lower value compared with real traces. This behavior suggests the presence of similar interest, i.e., people that go to same places. Observing the Rome trace (Fig. 9-(a)), we see that the value of topological overlap follows the traffic density. Also, at the rush times, we have more taxis sharing more neighbors. For the Helsinki trace (Fig. 9-(c)), we also observe the difference between the temporal and random graphs. Moreover, we observe a higher number of common neighbors' vehicles, which follows the features of the trace, i.e., groups of people going to the same place. Comparing the traces, Helsinki present major values than the Rome's trace.

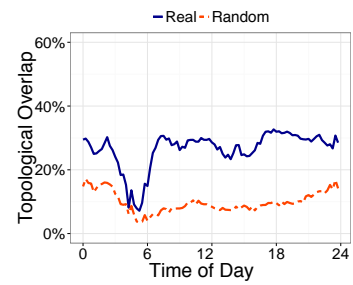

(a) Rome

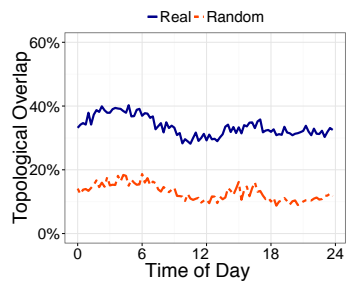

(b) Helsinki
Figure 9: Topological Overlap Daily evolution for Rome and Helsinki's traces.

To compute the edge frequency, we consider the encounter repetitiveness in each slot time during all of days. Besides, we also compare the results with the edge persistence to random graphs. Figure 10 shows the results for both traces. We can note that in Rome trace (Fig. 10-(a)) the edges are repeated on average $30 \%$ during the day. This happens due to the fact that people can go to the same place, but not necessarily they take the same taxi. However, the frequency value of 
random graphs is bigger than original traces, which describes the features of this type of vehicles. On contrast, in Helsinki trace (Fig. 10-(b)), the vehicles present a major value to persistence in rush hours close to $40 \%$, characterizing the people with similar behavior. Also, due to the characteristics of the working day model, this trace presents a major value of persistence than the random graphs, which defines social behaviors in this graph, i.e., the repetitiveness of behaviors.

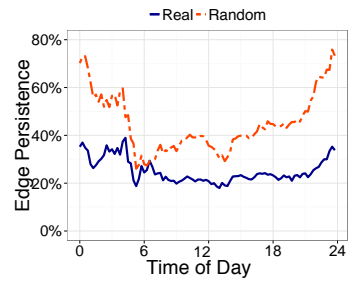

(a) Rome

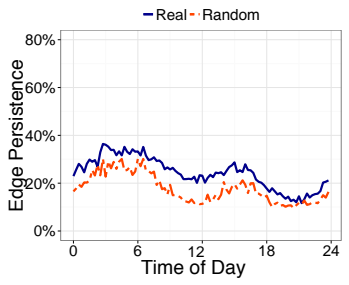

(b) Helsinki
Figure 10: Edge Persistence Daily evolution for Rome and Helsinki's traces.

\subsection{Metrics Correlation}

Aiming to verify the dependency level among the metrics and if there are metrics that portray the same behavior, we calculate the Pearson Correlation. This correlation defines the linear correlation between two variables. It defines the correlation level by a value, which ranges from -1 to 1 . When the value is positive there is a correlation, and we can define the level of correlation according to the scale: weak $(0,0$ to $\pm 0,3)$, moderate $( \pm 0,3$ to $\pm 0,7)$, and strong $( \pm 0,7$ to $\pm 1,0)[1]$.

Figure 11-(a) and (b) present the correlation results to the metrics topology overlap, clustering coefficient, node degree and betweenness to all traces analyzed. We observe that for both datasets the topology overlap and clustering coefficient present a higher correlation (0.9 to 1). Both metrics portray the presence of common interests among the vehicles, which explain this results. However, the correlation of these metrics and others is low. Another value observed is the correlation between node degree and betweenness. In fact, how higher is the degree of a node, higher will be the likelihood to more paths cross its, values explain range the interval 0.7 a 0.9 .

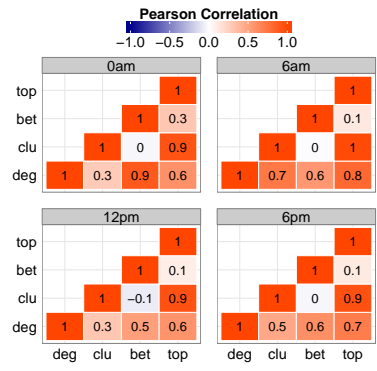

(a) Rome

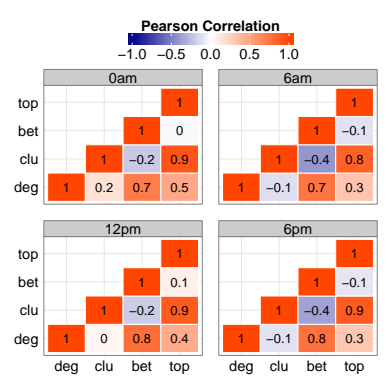

(b) Helsinki
Figure 11: Grid of Pearson Correlation between the metrics.

\section{USING INTERACTIONS}

The study of interactions in vehicular networks can open new perspectives. When considering the communication point of view, this study brings out new features to be considered in the design of communication protocols. For instance, by understanding the frequency of interactions it is possible to identify the best vehicles to perform a message broadcast, aiming to increase the message coverage and to reduce the message delivery delay and redundancy. For instance, in [3] we present a new solution to perform data dissemination by exploring interactions features.

Take into account the centrality of the vehicles, we can use this behavior to improve the data dissemination. The vehicles that portray higher centrality values are the best candidate to rebroadcast the data packets. When we reach these vehicles, the message can be propagated to the network quickly. In [15], the authors using these features to define the best dissemination scheme to use. Also, new strategies should be defined to reach the vehicles that present low centrality values. Probably, they are on the network border, presenting a low number of connections.

Considering the frequency of interactions, it can be classified as random or frequent. This property helps in identifying the vehicular interactions that are or not predicted. Thus, it is possible to identify the vehicles with same behaviors and routines, and then explore these vehicles to improve the routing process, for instance. Also, similar routines can be used to suggest places to visit, to promote carpooling services, to predict traffic jams, and to improve the mobility in the cities. In [21], the authors use these behaviors to establish stable vehicle groups, forming clusters in the network.

In the network design, the knowledge about the interactions can help to identify the places that have a higher probability of inducing new interactions, ensuring connectivity among vehicles. Thus, new RSU's placement strategies can be applied. Also, the vehicular density can be used to design new mechanisms to access the medium aware of this property, which contributes to improving the connectivity and the network throughput.

\section{CONCLUSION}

In this work, we presented a study of the interactions in vehicular networks using vehicular traces that describe the vehicular mobility. In this study, we use the Rome and Helsinki's trace. For both traces, we could verify high interactions of the people's routines on the vehicles' traffic. Despite Rome's traces being taxis vehicles, the passengers define the places to go. They share destinations and interests, forming communities on the graph. Besides, in Helsinki, we can also verify the influence of the people' desires in the traffic, with the presence of vehicles that share similar routines and behaviors.

Thus we can classify the interactions in Rome's Trace as acquaintance: a group of vehicles that share many common encounters, but not meeting often; and in Helsinki's Trace as friends: a group of vehicles that meet each other regularly and tend to know the same people. As future works, we intend to extend this evaluation grouping more traces that describe different mobility behaviors such as taxis, buses, cars, and bikes. Moreover, we will use more statistic tests, tools, and techniques in order to define mobility models that better portray the vehicles behaviors. Furthermore, we 
will use these results as an input to services and protocols in vehicular networks, aiming to improve the performance of services and to ameliorate the connectivity among the vehicles.

\section{ACKNOWLEDGMENTS}

The authors would like to thank CAPES, CNPq, FAPEMIG and INRIA for the financial support.

\section{REFERENCES}

[1] R. A. Becker, J. M. Chambers, and A. R. Wilks. The new s language. Pacific Grove, Ca.: Wadsworth 85 Brooks, 1988, 1, 1988.

[2] L. Bracciale, M. Bonola, P. Loreti, G. Bianchi, R. Amici, and A. Rabuffi. CRAWDAD data set roma/taxi (v. 2014-07-17). Downloaded from http://crawdad.org/roma/taxi/, July 2014.

[3] F. Cunha, A. Carneiro Viana, R. A. F. Mini, and A. A.F. Loureiro. Is it possible to find social properties in vehicular networks? In IEEE Symposium on Comp.and Com.(ISCC '14), 2014.

[4] F. D. Cunha, D. A. Alvarenga, A. C. Viana, R. A. Mini, and A. A. Loureiro. Understanding interactions in vehicular networks through taxi mobility. In Proceedings of the 12th ACM Symposium on Performance Evaluation of Wireless Ad Hoc, Sensor, E3\#38; Ubiquitous Networks, PE-WASUN '15, pages 17-24, New York, NY, USA, 2015. ACM.

[5] P. O. V. de Melo, A. C. Viana, M. Fiore, K. Jaffrès-Runser, F. L. Mouël, A. A. Loureiro, L. Addepalli, and C. Guangshuo. Recast: Telling apart social and random relationships in dynamic networks. Performance Evaluation, 87(0):19 - 36, 2015. Special Issue: Recent Advances in Modeling and Performance Evaluation in Wireless and Mobile Systems.

[6] F. Ekman, A. Keränen, J. Karvo, and J. Ott. Working day movement model. In Proceedings of the 1st ACM SIGMOBILE Workshop on Mobility Models, MobilityModels '08, pages 33-40, New York, NY, USA, 2008. ACM.

[7] M. Fiore and J. Härri. The networking shape of vehicular mobility. In ACM Int. Symposium on Mobile Ad Hoc Net. and Computing (MobiHoc '08), pages 261-272, 2008.

[8] T. Hossmann, T. Spyropoulos, and F. Legendre. Know thy neighbor: Towards optimal mapping of contacts to social graphs for dtn routing. In INFOCOM, 2010 Proceedings IEEE, pages 1-9, March 2010.

[9] N. L. Johnson and S. Kotz. Urn Models and Their Applications: An Approach to Modern Discrete Probability Theory. Wiley, New York, 1977.

[10] X. Liu, Z. Li, W. Li, S. Lu, X. Wang, and D. Chen. Exploring social properties in vehicular ad hoc networks. In Proceedings of the Fourth Asia-Pacific
Symposium on Internetware, Internetware '12, pages 24:1-24:7, New York, NY, USA, 2012. ACM.

[11] N. Loulloudes, G. Pallis, and M. D. Dikaiakos. The dynamics of vehicular networks in urban environments. CoRR, abs/1007.4106, 2010.

[12] S. Maaroufi and S. Pierre. Vehicular social systems: An overview and a performance case study. In Proceedings of the Fourth ACM International Symposium on Development and Analysis of Intelligent Vehicular Networks and Applications, DIVANet '14, pages 17-24, New York, NY, USA, 2014. ACM.

[13] G. Orman and V. Labatut. A comparison of community detection algorithms on artificial networks. In J. Gama, V. Costa, A. Jorge, and P. Brazdil, editors, Discovery Science, volume 5808 of Lecture Notes in Computer Science, pages 242-256. Springer Berlin Heidelberg, 2009.

[14] G. Pallis, D. Katsaros, M. Dikaiakos, N. Loulloudes, and L. Tassiulas. On the structure and evolution of vehicular networks. In Modeling, Analysis Simulation of Computer and Telecommunication Systems, 2009. MASCOTS '09. IEEE International Symposium on, pages $1-10$, Sept 2009.

[15] J. Sanguesa, M. Fogue, P. Garrido, F. J. Martinez, J.-C. Cano, C. T. Calafate, and P. Manzoni. On the selection of optimal broadcast schemes in vanets. In Proceedings of the 16th ACM International Conference on Modeling, Analysis 83\#38; Simulation of Wireless and Mobile Systems, MSWiM '13, pages 411-418, New York, NY, USA, 2013. ACM.

[16] F. A. Silva, C. Celes, A. Boukerche, L. B. Ruiz, and A. A. F. Loureiro. Filling the gaps of vehicular mobility traces. In The 18th ACM International Conference on Modeling, Analysis and Simulation of Wireless and Mobile Systems, 2015.

[17] S. Uppoor and M. Fiore. Insights on metropolitan-scale vehicular mobility from a networking perspective. In Proceedings of the 4th ACM international workshop on Hot topics in planet-scale measurement, HotPlanet '12, pages 39-44, New York, NY, USA, 2012. ACM.

[18] A. M. Vegni and V. Loscri. A survey on vehicular social networks. Communications Surveys 85 Tutorials, IEEE 17(4):2397-2419, 2015.

[19] D. J. Watts and S. H. Strogatz. Collective dynamics of 'small-world' networks. Nature, 393(6684):440-442, June 1998.

[20] B. Wellman. Computer networks as social networks. Science, 293(5537):2031-2034, 2001.

[21] Z. Zhang, A. Boukerche, and R. Pazzi. A novel multi-hop clustering scheme for vehicular ad-hoc networks. In Proceedings of the 9th ACM International Symposium on Mobility Management and Wireless Access, MobiWac '11, pages 19-26, New York, NY, USA, 2011. ACM. 